\title{
Perivascular epithelioid cell tumor (PEC-ome) of the prostate: Ultrasound feature in case report
}

\author{
Giulia Sbrollini $^{1,2}$, Alessandro Conti ${ }^{1,2}$, Andrea Benedetto Galosi ${ }^{1}$, Vito Lacetera ${ }^{2}$, \\ Rodolfo Montironi ${ }^{3}$, Lorenzo Montesi ${ }^{2}$, Giovanni Muzzonigro ${ }^{2}$ \\ ${ }^{1}$ Department of Urology, "Augusto Murri" General Hospital, ASUR Marche, Fermo, Italy; \\ ${ }^{2}$ Institute of Urology, Ospedali Riuniti, Politechnic University of the Marche, Ancona, Italy; \\ ${ }^{3}$ Institute of Pathology, Ospedali Riuniti, Politechnic University of the Marche, Ancona, Italy.
}

\begin{abstract}
Summary Introduction: We describe a rare tumor arising from the prostate gland: Perivascular Epithelioid Cells tumor (PEC-ome). A 54-years old was treated for acute urinary retention with alpha-blockers at presentation due to benign prostate enlargement $(65 \mathrm{cc})$ with asymmetric middle lobe and regular PSA $(0.92 \mathrm{ng} / \mathrm{ml})$. After 5 months, patient developed a second acute urinary retention episode and nodules in the left lung; he was treated with transurethral resection of the prostate and left lobectomy.

Results: Histological examination of prostate and lung tissue gave the same diagnosis: leiomyosarcoma with atypical morphological features and patient was observed for 4 months. Considering the uncommon diagnosis, pathological review by the uro-pathologist at our Hospital was done. Additional immunohistochemistry was done and both tumors showed similar and typical features of metastatic PEC-ome (T1b NO M1). Therefore a new staging showed local and distant progression with prostatic mass and small lung metastasis. Three cycles of Gemcitabine and Pazopanib were administered, but 2 months later a new urinary retention occurred, despite chemotherapy. Patient referred to our Hospital for salvage pelvic surgery with lymph node dissection. Final pathological diagnosis was PECome of the prostate stage pT4 pNO RO M1.

Conclusions: PEC-ome is a rare but rapidly invasive mesothelial tumor with early metastatic potential. When this tumors originates from the fibromuscular stroma of the prostate it mimics benign prostatic enlargement and causes LUTS. Expert pathology aided by immunoisthochemistry is the cornerstone of diagnosis. There are no pathognomonic imaging on ultrasound or symptoms suggesting the presence of PECome in early stage. A multidisciplinary approach is necessary and radical surgery should be done to treat this aggressive cancer.
\end{abstract}

KEY WORDS: Perivascular epithelioid cell neoplasms; Ultrasound; Prostate neoplasms; Diagnosis; Surgery.

Submitted 3 October 2014; Accepted 31 October 2014

\section{INTRODUCTION}

Perivascular Epithelioid Cell tumor (PEC-ome) is a perivascular epithelioid neoplasia, that is a mesothelial tumor (1-3).

Introduction, Figures and Conclusions are posted are posted in Supplemmentary Materials on www.aiua.it

\section{Case presentation}

We describe a case of a 53-yrs old man affected by PEComa of the prostate, presented with lung metastasis at the diagnosis. The patient was first hospitalized in December 2012 for an episode of acute urinary retention after a period of dysuria, nocturia and urinary symptoms. A urinary catheter was placed for a week. Chest Xray and renal ultrasonography performed at admission were negative. TRUS highlighted an increased prostate volume, not homogeneous ultrasound pattern with two solid nodular formations of $24 \mathrm{~mm}$, compatible with benign hyperplasia of the middle lobe, with large periurethral and parenchymal calcifications. The posterior profile of the gland, as well as the structure of the seminal vesicles appeared regular. PSA was always normal $($ PSA tot $=0.9 \mathrm{ng} / \mathrm{ml}$; PSA free $=0.2 \mathrm{ng} / \mathrm{ml})$. Four months later a second episode of acute urinary retention occurred and endoscopic transurethral resection of the prostate (TURP) was performed.

Preoperative chest X-ray emphasized the presence of a rounded lung opacity, confirmed by contrast-enhanced computed tomographyb (CT), that documented an ovoidshape solid nodule, with sharp margins $(40 \mathrm{~mm})$ and contemporary similar small imagines, bilaterally.

Histological analysis of the resected prostatic tissue showed cellular proliferation of partly spindled epithelioid elements with clear cytoplasm, marked irregular atypias, necrotic areas with high mitotic index with atypical mitoses.

IHC staining showed positivity for muscle-specific and connective tissue markers as desmin, caldesmonin, calponin, smooth-muscle actin and vimentine, orienting the diagnosis to a grade IV leiomyosarcome.

Nevertheless, contemporary positivity for some epithe- 
lial markers as Citokeratin 18, CAM5.2, EMA, as far as CD10 posed the differential diagnosis with a metastasis of sarcomatoid clear cell renal cell carcinoma.

After a total body positron-emission tomography (PET)CT scan that confirmed the metabolically active nature of the nodule (SUV max 5.38) and the thoracic Surgeon performed in a left lower pulmonary lobectomy. Histological examination of pulmonary mass showed a tumor with large cells characterized from a clear cytoplasm, severe atypical cyto-morphology and high mitotic activity. In consideration of the similarities between the two pathological features, the urologist submitted the case along with the respective histological samples (lung and prostate) to an expert uro-pathologist (RM), who confirmed the morphological and IHC similarity between pulmonary and prostatic lesions, with the constant presence of cells with clear cytoplasm and polymorphic nuclei and, to a lesser degree, of spindle cells. The prostate specimen showed an infiltrative appearance of the tumor, with a IHC positivity for smooth muscle-specific actin, focal HMB45 and MelanA, as well as positivity for TFE3 (Figure 1). It leads to the definitive confirmation of the diagnosis of PEC-ome with prostatic and pulmonary localization at the onset, T1b N0 M1. Molecular study of the specimens, performed at the John Hopkins Institute, permitted to further support tumor identification.

Staging total body CT scan, after 4 months without treatment, showed an increase in the number and size of some of the pulmonary lesions and some lymph nodes enlargements in the mediastinum ( $\max : 1.1 \mathrm{~cm}$ ). The residual prostate appeared increased in size, elevating the bladder floor. It finally ruled out the renal nature of the tumor. After oncological evaluation, indication to 3 cycles chemotherapy (CTX) with Gemcitabine was given.

Two months CT scan, during CTX treatment, showed progression with solid tissue of the prostate $(7 \times 5.4 \mathrm{~cm})$ projecting into the lumen of the bladder. Prostate tissue appeared to be infiltrating the bladder walls. All the documentation of the case was sent for counseling to the national referring center for tumors (IRCCS, Milano, Director Roberto Salvioni). Considering also the discomfort associated with the presence of the indwelling bladder catheter, it was held for reasonable to propose the patient an intervention of cystoprostatectomy. The patient was then referred to our hospital for cystoprostatectomy with extended lymphadenectomy.

A control TRUS performed preoperatively, confirmed, in B mode, dyshomogeneous periurethral tissue, with diffuse calcifications and a hypo-anechoic nodular formation of the anterior portion of the prostate. The margins in the peripheral zone of the gland were apparently safe, failing to show any sonographic signs of infiltration of the pelvic structures surrounding the bladder (Figure 2-a).

The power-Doppler mode let us see a massive vascularization, with the presence of a central artery, going from the apex of the prostate to the bladder neck, spraying the tumor mass itself (Figure 2-b).

The postoperative course was uneventful. The definitive histological examination confirmed the diagnosis of prostate PEC-oma, infiltrating the bladder, pT4 pN0 R0 M1. The surgical margins and all the 39 lymph nodes removed were free from the tumor. Complementary therapy with Pazopanib was then performed.

In the diagnosis of malignant prostate tumors, a series of diseases with extremely low incidence but significantly unfavorable prognosis should be considered in addition to the most common histology of adenocarcinoma. Although the initial diagnostic path is difficult, imaging techniques, if properly interpreted, can provide elements that address the clinical suspicion of an unusual variant. Nevertheless, a final guideline is still missing and the possibility of early treatment is left to the skill and intuition of the clinician and the expertise of the pathologist.

\section{REFERENCES}

1. Vang R, Kempson RL. Perivascular epithelioid cell tumor ('PEComa') of the uterus: a subset of HMB-45-positive epithelioid mesenchymal neoplasms with an uncertain relationship to pure smooth muscle tumors. Am J Surg Pathol. 2002; 26:1-13.

2. Folpe AL. Neoplasms with perivascular epithelioid cell differentiation (PEComas). In: Fletcher CDM, Unni KK, Epstein J, Mertens F (eds) Pathology and genetics of tumours of soft tissue and bone. Series: WHO Classification of tumours. IARC Press, Lyon, 2002; pp 221-222.

3. Martignoni G, Pea M, Reghellin D, et al. PEComas: the past, the present and the future. Virchows Arch. 2008; 452:119-132.

\author{
Correspondence \\ Giulia Sbrollini, MD, Resident in urology (Corresponding Author) \\ giuliasbrollini@libero.it \\ Alessandro Conti, MD, Urologist \\ alessandro.conti@hotmail.com \\ Vito Lacetera, MD \\ Lorenzo Montesi, MD, Resident in Urology \\ lorenzomontesi@yahoo.it \\ Giovanni Muzzonigro, MD, Urologist \\ g.muzzonigro@univpm.it \\ Rodolfo Montironi, MD, Pathologist \\ r.montironi@univpm.it \\ AOU United Hospitals Polytechnic University of Marche Region, \\ Ancona, Italy
}

Andrea Benedetto Galosi, MD, Urologist

galosiab@yahoo.it

Department of Urology, "Augusto Murri" General Hospital, ASUR Marche, Fermo, Italy 